\title{
THE $\infty$-BESOV CAPACITY PROBLEM
}

\author{
M. MILMAN AND J. XIAO
}

\begin{abstract}
A theory of $\infty$-Besov capacities is developed and several applications are provided. In particular, we solve an open problem in the theory of limits of the $\infty$-Besov semi-norms, we obtain new restriction-extension inequalities, and we characterize the point-wise multipliers acting on the $\infty$-Besov spaces.
\end{abstract}

\section{INTRODUCTION}

In this paper (cf. Section 2 below) we introduce a new 1 theory of capacities and perimeters associated to the Besov spaces $\Lambda_{\alpha}^{p, q}$, with parameters $(\alpha, p, q) \in(0,1) \times[1, \infty] \times[1, \infty]$, with particular emphasis on the case $q=\infty$ (the $\infty$-Besov spaces). Our theory has interesting applications: In Section 3 we apply it to characterize the restrictions and extensions of the $\infty$ Besov functions, and in Section 4, we provide a characterization of the point-wise multipliers for the $(\alpha, p, \infty)$-Besov spaces. There are, of course, many other interesting connections. For example, we mention that the corresponding spaces of traces are naturally linked to the theory of function spaces based on outer measures that was recently developed in [9] (cf. Section 3). Moreover, and somewhat surprisingly, the $\infty$-Besov spaces can be embedded in the Campanato spaces (cf. Remark 2 below).

Another interesting application occurs when dealing with an open end point problem in the theory of limits of Besov or fractional Sobolev norms. We shall now develop this application in some detail, as we believe it offers a nice application and an introduction to some of underlying issues dealt with in this paper.

The limiting inequalities we intend to extend originated in applications to PDEs, and were considered by a number of authors (cf. [4], [5], [22], [23], [19]). For example, BourgainBrezis-Mironescu [5] show that

$$
\lim _{\alpha \rightarrow 1}(1-\alpha)\|f\|_{\dot{\Lambda}_{\alpha}^{p, p}}^{p}=\lim _{\alpha \rightarrow 1}(1-\alpha) \int_{\mathbb{R}^{n}} \int_{\mathbb{R}^{n}} \frac{|f(x)-f(y)|^{p}}{|x-y|^{n+\alpha p}} d x d y=\left(\int_{\mathbb{S}^{n-1}}|\cos \theta|^{p} d \sigma\right)\|\nabla f\|_{L^{p}}^{p},
$$

where $p \in[1, \infty), \mathbb{S}^{n-1}$ is the unit sphere of $\mathbb{R}^{n \geq 1}, \theta$ is the angle derivation from the vertical, and $d \sigma$ is the standard surface area measure. In [22, 23] Maz'ya-Shaposhnikova show

$$
\lim _{\alpha \rightarrow 0} \alpha\|f\|_{\Lambda_{\alpha}^{p, p}}^{p}=\lim _{\alpha \rightarrow 0} \alpha \int_{\mathbb{R}^{n}} \int_{\mathbb{R}^{n}} \frac{|f(x)-f(y)|^{p}}{|x-y|^{n+\alpha p}} d x d y=2 p^{-1} \sigma_{n-1}\|f\|_{L^{p}}^{p},
$$

2010 Mathematics Subject Classification. 31C15, 35J92, 42B37, 46E35.

MM was partially supported by a grant from the Simons Foundation (\#207929 to Mario Milman), JX is in part supported by NSERC of Canada.

${ }^{1}$ The theory builds from previous works (cf. [1, 2, 32, 34, 35, 36, 31, 8]). 
where $p \in[1, \infty)$, and $\sigma_{n-1}$ denotes the surface measure of $\mathbb{S}^{n-1}$. A related inequality obtained in [5], and sharpened in [22], can be formulated as

$$
\|f\|_{L^{\frac{p n}{n-\alpha p}}} \leq\left(c(n, p) \alpha(1-\alpha)(n-\alpha p)^{1-p}\right)^{\frac{1}{p}}\|f\|_{\dot{\Lambda}_{\alpha}^{p, p}},
$$

where $(\alpha, p) \in(0,1) \times[1, n / \alpha)$ and $c(n, p)$ is a constant depending only on $n$ and $p$. To derive each of these results required a new understanding about fractional norms?

From a more general point of view, the Besov spaces $\Lambda_{\alpha}^{p, q}$ can be also obtained by real interpolation and, as it turns out, the limiting formulae above can be also understood in the more general setting of interpolation theory (cf. [24]). In particular, this point of view led us to extend these limiting theorems to higher order norms (cf. [16]). Further results in this direction have been also obtained by Triebel [29].

A natural question that has remained open is the characterization of the limits of the homogeneous Besov norms $\|\cdot\|_{\Lambda_{\alpha}^{p, q}}$ that correspond to the choices $p=\infty$ or $q=\infty$. As it is well known, the $\infty$-Besov spaces $\Lambda_{\alpha}^{p, \infty}$ are connected to the Sobolev spaces. Indeed, the if $(\alpha, p) \in\{1\} \times(1, \infty)$ then $f$ is in the first-order Sobolev $p$-space $W^{1, p}$ if and only if $f \in L^{p}$ and $\sup _{h \in \mathbb{R}^{n}}|h|^{-1}\left\|\Delta_{h} f\right\|_{L^{p}}<\infty$ (cf. [37, Theorem 2.16]), moreover, if $\alpha=1=p$, then $f$ is of bounded variation on $\mathbb{R}^{n}$, i.e. $f \in B V$, if and only if $f \in L^{1}$ and $\sup _{h \in \mathbb{R}^{n}}|h|^{-1}\left\|\Delta_{h} f\right\|_{L^{1}}<\infty$ (cf. [7, p. 245]).

Returning to the limiting theorems above, let us now show how our theory of capacities can be used to add a new end point result to the Bourgain-Brezis-Mironescu-Maz'yaShaposhnikova formulae. Let $\|\cdot\|_{B V}$ denote the standard BV-norm, and let

$$
P_{1,1, \infty}\left(\left\{x \in \mathbb{R}^{n}: f(x)>t\right\}\right)=\left\|1_{\{f>t\}}(\cdot)\right\|_{\dot{\Lambda}_{\alpha}^{1, \infty}}
$$

(cf. Section 2). Suppose that $f \in B V\left(\mathbb{R}^{n}\right)$ and let $\alpha \in(0,1)$. Since $f \in L^{1}\left(\mathbb{R}^{n}\right)$, we have (cf. Proposition 3(1) and (3) below for more details) that, for each $h \in \mathbb{R}^{n}$,

$$
\begin{aligned}
|h|^{-1}\left\|\Delta_{h} f\right\|_{L^{1}} & \leq \int_{0}^{\infty}|h|^{-1}\left\|1_{\{f>t\}}(\cdot+h)-1_{\{f>t\}}(\cdot)\right\|_{L^{1}} d t \\
& \leq \int_{0}^{\infty}|h|^{-1}|h| P_{1,1, \infty}\left(\left\{x \in \mathbb{R}^{n}: f(x)>t\right\}\right) d t \\
& \approx\|f\|_{B V} .
\end{aligned}
$$

Let us write

$$
\|f\|_{\dot{\Lambda}_{\alpha}^{\alpha, \infty}} \leq \sup _{|h| \leq 1}|h|^{-\alpha}\left\|\Delta_{h} f\right\|_{L^{1}}+\sup _{|h|>1}|h|^{-\alpha}\left\|\Delta_{h} f\right\|_{L^{1}}
$$

Let $\epsilon>0$. Then we can find non-zero $h_{1}:=h_{1}(\alpha) \in \mathbb{R}^{n}$, with $\left|h_{1}\right| \leq 1$, such that

$$
\|f\|_{\Lambda_{\alpha}^{1, \infty}}<\left|h_{1}\right|^{-\alpha}\left\|\Delta_{h_{1}} f\right\|_{L^{1}}+\sup _{|h|>1}|h|^{-\alpha}\left\|\Delta_{h} f\right\|_{L^{1}}+\epsilon .
$$

The first term in the last inequality can be estimated by

$$
\begin{aligned}
\left|h_{1}\right|^{-\alpha}\left\|\Delta_{h_{1}} f\right\|_{L^{1}} & \leq\left|h_{1}\right|^{1-\alpha}\left|h_{1}\right|^{-1}\left\|\Delta_{h_{1}} f\right\|_{L^{1}} \\
& \leq\left|h_{1}\right|^{-1}\left\|\Delta_{h_{1}} f\right\|_{L^{1}} \\
& \lesssim\|f\|_{B V} .
\end{aligned}
$$

\footnotetext{
${ }^{2}$ See also Remark 5(ii) below.
} 
Moreover, using the mean value theorem and noting that the function $t \mapsto t^{\alpha} \ln t$ is bounded on $[0,1]$, we see that $\lim _{\alpha \rightarrow 1} t^{\alpha}=t$, uniformly on $[0,1]$, thus

$$
\limsup _{\alpha \rightarrow 1} \sup _{|h|>1}|h|^{-\alpha}\left\|\Delta_{h} f\right\|_{L^{1}} \leq \sup _{|h|>1}|h|^{-1}\left\|\Delta_{h} f\right\|_{L^{1}} \lesssim\|f\|_{B V} .
$$

Collecting estimates, we see that

$$
\limsup _{\alpha \rightarrow 1}\|f\|_{\Lambda_{\alpha}^{1, \infty}} \lesssim\|f\|_{B V}+\epsilon .
$$

Consequently, letting $\epsilon \rightarrow 0$, we obtain

$$
\limsup _{\alpha \rightarrow 1}\|f\|_{\Lambda_{\alpha}^{1, \infty}} \lesssim\|f\|_{B V} .
$$

Notation. In the above and below, $A \approx B$ means $A \lesssim B \lesssim A$; while $A \lesssim B$ stands for $A \leq c B$ for a constant $c>0$.

\section{2. $\infty$-Besov SPACES AND CAPACITIES}

2.1. Besov spaces. The Besov spaces $\Lambda_{\alpha}^{p, q}\left(\mathbb{R}^{n}\right) \equiv \Lambda_{\alpha}^{p, q}$ that we shall consider in this paper will be defined in terms of difference operators (cf. e.g. [28, 21]). Let $h \in \mathbb{R}^{n}$, the difference operator, $\Delta_{h}$, acting on functions $f$ defined on $\mathbb{R}^{n}$, is given by,

$$
\Delta_{h} f(x)=f(x+h)-f(x) \quad \forall \quad x \in \mathbb{R}^{n} .
$$

Definition 1. The $\Lambda_{\alpha}^{p, q}$ spaces are defined according to the values of the parameters $(\alpha, p, q)$ as follows:

(i) If $(\alpha, p, q) \in(0,1) \times[1, \infty] \times[1, \infty)$, then $\Lambda_{\alpha}^{p, q}$ is the class of all $L^{p}$-functions $f$ such that

$$
\|f\|_{\Lambda_{\alpha}^{p, q}}=\left(\int_{\mathbb{R}^{n}}\left\|\Delta_{h} f\right\|_{L^{p}}^{q}|h|^{-(n+\alpha q)} d h\right)^{\frac{1}{q}}<\infty .
$$

(ii) If $(\alpha, p, q) \in(0,1) \times[1, \infty] \times\{\infty\}$ then $\Lambda_{\alpha}^{p, q}$ is the class of all $L^{p}$-functions $f$ such that

$$
\|f\|_{\dot{\Lambda}_{\alpha}^{p, q}}=\sup _{h \in \mathbb{R}^{n}}\left\|\Delta_{h} f\right\|_{L^{p}}|h|^{-\alpha}<\infty .
$$

For perspective, two interesting and important remarks on Definition 1 are given below to show further connections of the $\infty-$ Besov spaces with some classical function spaces.

Remark 1. Two comments on Definition \(i) are in order.

(i) It is interesting to observe that there is a natural Leibniz rule associated to $\|\cdot\|_{\Lambda_{\alpha}^{p, q} \text {, }}$ which is connected with a family of BMO-based Besov spaces extending the case $p=\infty$ of Definition 1 (i) due to $L^{\infty} \subset B M O$; see also Remark 2 (ii) . Let

$$
\|f\|_{\dot{\Lambda}_{\alpha}^{B M O, q}}=\left\{\begin{array}{l}
\left(\int_{\mathbb{R}^{n}}\left\|\Delta_{h} f\right\|_{B M O}^{q}|h|^{-(n+\alpha q)} d h\right)^{\frac{1}{q}} \text { as } q \in[1, \infty) ; \\
\sup _{h \in \mathbb{R}^{n}}\left\|\Delta_{h} f\right\|_{B M O}|h|^{-\alpha} \text { as } q=\infty .
\end{array}\right.
$$

Then (cf. [20] and more recently [25, (1.22)]), if $p \in(1, \infty]$ and $h \in \mathbb{R}^{n}$, we have

$$
\begin{aligned}
\left\|\Delta_{h}(f g)\right\|_{L^{p}} & =\|f(x+h) g(x+h)-f(x) g(x)\|_{L^{p}} \\
& =\left\|\Delta_{h} f(\cdot) g(\cdot+h)+f(\cdot) \Delta_{h} g(\cdot)\right\|_{L^{p}} \\
& \leq\left\|\Delta_{h} f(\cdot) g(\cdot+h)\right\|_{L^{p}}+\left\|f \Delta_{h} g\right\|_{L^{p}}
\end{aligned}
$$




$$
\begin{aligned}
& \leq\left\|\Delta_{h} f\right\|_{L^{p}}\|g(\cdot+h)\|_{B M O}+\left\|\Delta_{h} f\right\|_{B M O}\|g(\cdot+h)\|_{L^{p}}+\|f\|_{L^{p}}\left\|\Delta_{h} g\right\|_{B M O}+\|f\|_{B M O}\left\|\Delta_{h} g\right\|_{L^{p}} \\
& =\left\|\Delta_{h} f\right\|_{L^{p}}\|g\|_{B M O}+\left\|\Delta_{h} f\right\|_{B M O}\|g\|_{L^{p}}+\|f\|_{L^{p}}\left\|\Delta_{h} g\right\|_{B M O}+\|f\|_{B M O}\left\|\Delta_{h} g\right\|_{L^{p}}
\end{aligned}
$$

where we have used Minkowski's inequality and the translation-invariance of $\|\cdot\|_{L^{p}}$ and $\|\cdot\|_{B M O}$. Consequently,

$\|f g\|_{\hat{\Lambda}_{\alpha}^{p, q}} \lesssim\|f\|_{\hat{\Lambda}_{\alpha}^{p, q}}\|g\|_{B M O}+\|g\|_{\dot{\Lambda}_{\alpha}^{p, q}}\|f\|_{B M O}+\|f\|_{L^{p}}\|g\|_{\dot{\Lambda}_{\alpha}^{B M O, q}}+\|f\|_{\dot{\Lambda}_{\alpha}^{B M O, q}}\|g\|_{L^{p}}$

$$
\lesssim\left(\|f\|_{\Lambda_{\alpha}^{p, q}}+\|f\|_{L^{p}}\right)\left(\|g\|_{B M O}+\|g\|_{\dot{\Lambda}_{\alpha}^{B M O, q}}\right)+\left(\|g\|_{\Lambda_{\alpha}^{p, q}}+\|g\|_{L^{p}}\right)\left(\|f\|_{B M O}+\|f\|_{\dot{\Lambda}_{\alpha}^{B M O, q}}\right) .
$$

(ii) Let

$$
I_{\alpha} g=\mathcal{F}^{-1}\left(|\zeta|^{-\alpha} \hat{g}(\zeta)\right)
$$

be the $\alpha$-Riesz potential of $g$ defined via the Fourier transform $\hat{g}=\mathcal{F} g$ and the inverse Fourier transform $\mathcal{F}^{-1}$. Moreover, let

$$
\|f\|_{I_{\alpha}(B M O)}=\|g\|_{B M O} \quad \text { if } \quad f=I_{\alpha} g .
$$

Let $1<q_{1}<2<q_{2}<\infty$. Then, the following implications hold for $\dot{\Lambda}_{\alpha}^{\infty, q}$ (cf. [30, Theorem 3.4]):

$$
\begin{aligned}
\|f\|_{\Lambda_{\alpha}^{\infty, 1}}<\infty & \Rightarrow\|f\|_{\Lambda_{\alpha}^{\infty, q_{1}}}<\infty \\
& \Rightarrow\|f\|_{\Lambda_{\alpha}^{\infty, 2}}<\infty \\
& \Rightarrow\|f\|_{I_{\alpha}(B M O)}<\infty \text { or }\|f\|_{\dot{\Lambda}_{\alpha}^{\infty, q_{2}}}<\infty \\
& \Rightarrow\|f\|_{\Lambda_{\alpha}^{\infty, \infty}}<\infty .
\end{aligned}
$$

Remark 2. Two comments on Definition \(ii) are in order:

(i) From [37, Theorem 2.16] and [7, p. 245] it follows that $f$ is in the first-order Sobolev $p$-space $W^{1, p}$ if and only if

$$
f \in L^{p} \quad \& \quad \sup _{h \in \mathbb{R}^{n}}|h|^{-1}\left\|\Delta_{h} f\right\|_{L^{p}}<\infty,
$$

and $f$ is of bounded variation on $\mathbb{R}^{n}$, i.e. $f \in B V$, if and only if

$$
f \in L^{1} \quad \& \quad \sup _{h \in \mathbb{R}^{n}}|h|^{-1}\left\|\Delta_{h} f\right\|_{L^{1}}<\infty .
$$

So, for $(\alpha, p) \in(0,1) \times[1, \infty)$, the $\infty$-Besov space $\Lambda_{\alpha}^{p, \infty}$ can be treated as the fractional extensions of $W^{1, p}$ and $B V$.

(ii) Although the inclusions

$$
\dot{\Lambda}_{\alpha}^{p, q} \subset \dot{\Lambda}_{\alpha}^{p, \infty},(\alpha, p, q) \in(0,1) \times[1, \infty) \times[1, \infty),
$$

are well-known, it is quite surprising that for $(\alpha, p) \in(0,1) \times[1, \infty)$ the homogeneous $\infty$-Besov space $\dot{\Lambda}_{\alpha}^{p, \infty}$ embeds in the family of Campanato spaces. Towards a proof of this fact this let $\delta>0$ and $(\alpha, p) \in(0,1) \times[1, \infty)$; then if $f \in \dot{\Lambda}_{\alpha}^{p, \infty}$ we have

$$
\int_{|h|<\delta}\left\|\Delta_{h} f\right\|_{L^{p}}^{p} d h \lesssim\|f\|_{\dot{\Lambda}_{\alpha}^{p, \infty}}^{p} \delta^{\alpha p+n}
$$

Consequently,

$$
\int_{\left|x-x_{0}\right|<\frac{\delta}{2}} \int_{\left|y-x_{0}\right|<\frac{\delta}{2}}|f(x)-f(y)|^{p} d y d x \lesssim\|f\|_{\dot{\Lambda}_{\alpha}^{p, \infty}}^{p} \delta^{\alpha p+n} .
$$


Let $B\left(x_{0}, r\right)$ be the Euclidean ball with center $x_{0}$, radius $r$, and Lebesgue measure $\left|B\left(x_{0}, r\right)\right|$. Let $f_{E}$ stand for the integral mean of $f$ over $E \subset \mathbb{R}^{n}$, with respect to the Lebesgue measure $d x$ or $d y$. Using Jensen's inequality we readily obtain

$$
\int_{B\left(x_{0}, \frac{\delta}{2}\right)} \int_{B\left(x_{0}, \frac{\delta}{2}\right)}|f(x)-f(y)|^{p} d y d x \geq\left|B\left(x_{0}, \frac{\delta}{2}\right)\right| \int_{B\left(x_{0}, \frac{\delta}{2}\right)}\left|f(x)-f_{B\left(x_{0}, \frac{\delta}{2}\right)} f\right|^{p} d x,
$$

thus we see that

$$
\|f\|_{p, \alpha}=\sup _{\left(x_{0}, r\right) \in \mathbb{R}^{n} \times(0, \infty)} r^{-\alpha}\left\|f-f_{B\left(x_{0}, r\right)} f\right\|_{L^{p}\left(B\left(x_{0}, r\right)\right)} \lesssim\|f\|_{\Lambda_{\alpha}^{p, \infty}} .
$$

We note the following consequences of the previous discussion:

- If $\alpha p<n$, then $\|f\|_{\Lambda_{\alpha}^{p, \infty}}<\infty$, implies that $f$ belongs to the $\left(-\alpha+\frac{n}{p}\right)$-Campanato class $\mathcal{L}^{p, \alpha p}$, i.e., $\|f\|_{\mathcal{L}^{p, \alpha p}}=\|f\|_{p, \alpha}<\infty$; cf. [11, p.67].

- If $\alpha p=n$, then $\|f\|_{\Lambda_{\alpha}^{p, \infty}}<\infty$ implies that f belongs to the class BMO offunctions with bounded mean oscillation, i.e., $\|f\|_{B M O}=\|f\|_{p, \frac{n}{p}}<\infty$; $c f$. [14].

- If $\alpha p>n$, then $\|f\|_{\Lambda_{\alpha}^{p, \infty}}<\infty$ implies that $f$ is $\left(\alpha-\frac{n}{p}\right)$-Hölder-continuous, i.e., $\|f\|_{\Lambda_{\alpha-\frac{n}{p}}^{\infty, \infty}} \approx\|f\|_{p, \alpha}<\infty ; c f$. [11, p.70].

2.2. $\infty$-Besov capacities. Motivated by [13, p.27] and Remark 2(ii), we introduce the following definition.

Definition 2. Let $C\left(\mathbb{R}^{n}\right)$ be the class of all continuous functions on $\mathbb{R}^{n}$. Then the (homogeneous) $\infty$-Besov capacity of a set $E \subset \mathbb{R}^{n}$ is defined by

$$
C_{\alpha, p, q}(E)=\left\{\begin{array}{lll}
\inf \left\{\|f\|_{\dot{\Lambda}_{\alpha}^{p, \infty}}^{p}: f \in \mathrm{A}_{\alpha, p, \infty}(E)\right\} & \text { as } & (\alpha, p, q) \in(0,1) \times[1, \infty] \times\{\infty\} ; \\
\inf \left\{\|f\|_{\dot{\Lambda}_{\alpha}^{\infty, q}}^{q}: f \in \mathrm{A}_{\alpha, \infty, q}(E)\right\} & \text { as } & (\alpha, p, q) \in(0,1) \times\{\infty\} \times[1, \infty]
\end{array}\right.
$$

where

$$
\mathrm{A}_{\alpha, p, q}(E)=\left\{f \in \Lambda_{\alpha}^{p, q} \cap C\left(\mathbb{R}^{n}\right): f \geq 1_{N} \text { on a neighbourhood } N \text { of } E\right\}
$$

and $1_{N}$ is the indicator of $N$.

We shall now explore the nature of the $\infty$-Besov capacities.

Proposition 1. Suppose $(\alpha, q) \in(0,1) \times[1, \infty]$. Then $C_{\alpha, \infty, q}(E)=0 \forall E \subset \mathbb{R}^{n}$.

Proof. The result follows immediately from the fact that constant functions belong to $\Lambda_{\alpha}^{\infty, q} \cap$ $C\left(\mathbb{R}^{n}\right)$, when $q \in[1, \infty]$.

Consequently, the interesting situation of $C_{\alpha, p, q}(\cdot)$ is the capacity $C_{\alpha, p, \infty}$ under $(\alpha, p) \in$ $(0,1) \times[1, \infty)$. Referring to [18, 8], we have the following basic properties.

Proposition 2. Let $(\alpha, p) \in(0,1) \times[1, \infty)$. Then:

(1) $C_{\alpha, p, \infty}(\emptyset)=0$.

(2) $C_{\alpha, p, \infty}\left(E_{1}\right) \leq C_{\alpha, p, \infty}\left(E_{2}\right)$ whenever $E_{1} \subseteq E_{2}$.

(3) $C_{\alpha, p, \infty}\left(E_{1} \cup E_{2}\right) \leq C_{\alpha, p, \infty}\left(E_{1}\right)+C_{\alpha, p, \infty}\left(E_{1}\right)$ provided $E_{1}, E_{2} \subset \mathbb{R}^{n}$.

(4) $C_{\alpha, p, \infty}(E)=\inf \left\{C_{\alpha, p, \infty}(O):\right.$ open $\left.O \supseteq E\right\}$.

(5) $C_{\alpha, p, \infty}\left(\cap_{j=1}^{\infty} K_{j}\right)=\lim _{j \rightarrow \infty} C_{\alpha, p, \infty}\left(K_{j}\right)$, where the $K_{j}^{\prime}$ s are compact subsets of $\mathbb{R}^{n}$, with $K_{j} \supseteq K_{j+1} \forall j=1,2,3, \ldots$ 
Proof. (1) and (2) follow immediately from the definition of $C_{\alpha, p, \infty}(\cdot)$.

(3) For any given $\epsilon>0, j=1,2$, pick $f_{j} \in \mathrm{A}_{\alpha, p, \infty}\left(E_{j}\right)$ such that

$$
\left\|f_{j}\right\|_{\dot{\Lambda}_{\alpha}^{p, \infty}}^{p}<C_{\alpha, p, \infty}\left(E_{j}\right)+\epsilon .
$$

Note that

$$
\left\{\begin{array}{l}
f=\max \left\{f_{1}, f_{2}\right\} \in \mathrm{A}_{\alpha, p, \infty}\left(E_{1} \cup E_{2}\right) \\
\left\|\Delta_{h} f\right\|_{L^{p}}^{p} \leq\left\|\Delta_{h} f_{1}\right\|_{L^{p}}^{p}+\left\|\Delta_{h} f_{2}\right\|_{L^{p}}^{p}
\end{array}\right.
$$

Consequently,

$$
C_{\alpha, p, \infty}\left(E_{1} \cup E_{2}\right) \leq\|f\|_{\dot{\Lambda}_{\alpha}^{p, \infty}}^{p} \leq C_{\alpha, p, \infty}\left(E_{1}\right)+C_{\alpha, p, \infty}\left(E_{2}\right)+2 \epsilon .
$$

Letting $\epsilon \rightarrow 0$ the desired result follows.

(4) In view of (2), the verification of (4) will be complete once we prove

$$
C_{\alpha, p, \infty}(E) \geq \inf \left\{C_{\alpha, p, \infty}(O): \text { open } O \supseteq E\right\} .
$$

Now, for any $\epsilon>0$ there exists a function $f_{0} \in \mathrm{A}_{\alpha, p, \infty}(E)$, a neighborhood $O$ of $E$ such that $f_{0} \geq 1$ in $O$, and, moreover,

$$
\left\|f_{0}\right\|_{\dot{\Lambda}_{\alpha}^{p, \infty}}^{p}<C_{\alpha, p, \infty}(E)+\epsilon
$$

But since we also have

$$
C_{\alpha, p, \infty}(O) \leq\left\|f_{0}\right\|_{\dot{\Lambda}_{\alpha}^{p, \infty}}^{p},
$$

the required inequality follows from combining the last two inequalities and letting $\epsilon$ go to 0 .

(5) Suppose that $K_{1} \supseteq K_{2} \supseteq K_{3} \cdots \supseteq K=\cap_{j=1}^{\infty} K_{j}$ is a sequence of compact subsets of $\mathbb{R}^{n}$. Then $\left\{C_{\alpha, p, \infty}\left(K_{j}\right)\right\}$ is a decreasing numerical sequence and therefore has a limit as $j \rightarrow \infty$. Let $O$ be any open set such that $O \supset K$. Since $K$ is compact, there exists an index $j$ such that $K_{j} \subset O$, whence,

$$
\lim _{j \rightarrow \infty} C_{\alpha, p, \infty}\left(K_{j}\right) \leq C_{\alpha, p, \infty}(O) .
$$

The last estimate, combined with (2)\&(4), implies

$$
C_{\alpha, p, \infty}(K) \leq \lim _{j \rightarrow \infty} C_{\alpha, p, \infty}\left(K_{j}\right) \leq C_{\alpha, p, \infty}(K) .
$$

Remark 3. We have been unable to prove that $C_{\alpha, p, \infty}$ is countably subadditive, but refer the interested reader to [17, 18, 12] for a discussion on the so-called Sobolev capacity and BV capacity on metric spaces.

Proposition 3. Let $(\alpha, \beta, p) \in(0,1) \times(0, \infty) \times[1, \infty)$. For $E \subset \mathbb{R}^{n}$ let

$\circ E-h=\{x-h: x \in E\}$ be its $\mathbb{R}^{n} \ni$ h-left translation;

○

$$
P_{\alpha, p, \infty}(E)=\left\|1_{E}\right\|_{\Lambda_{\alpha}^{p, \infty}}=\sup _{h \in \mathbb{R}^{n}}|h|^{-\alpha}(2(|E|-|E \cap(E-h)|))^{\frac{1}{p}}
$$

be its $(\alpha, p, \infty)$-perimeter;

$$
H^{\beta}(E)=\inf \left\{\sum_{j=1}^{\infty}\left(\frac{\pi^{\frac{\beta}{2}}}{\Gamma\left(1+\frac{\beta}{2}\right)}\right) r_{j}^{\beta}: E \subseteq \cup_{j=1}^{\infty} B\left(x_{j}, r_{j}\right)\right\}
$$

be its $\beta$-dimensional Hausdorff capacity with $\Gamma(\gamma)=\int_{0}^{\infty} e^{-t} t^{\gamma-1}$ and $\gamma \in(0, \infty)$. 
(1) Suppose $f \in \Lambda_{\alpha}^{p, \infty} \cap C\left(\mathbb{R}^{n}\right)$. Then,

$$
\left(t^{p} C_{\alpha, p, \infty}\left(\left\{x \in \mathbb{R}^{n}:|f(x)|>t\right\}\right)\right)^{\frac{1}{p}} \leq\|\mid f\|_{\dot{\Lambda}_{\alpha}^{p, \infty}} \leq \int_{0}^{\infty} P_{\alpha, p, \infty}\left(\left\{x \in \mathbb{R}^{n}:|f(x)|>t\right\}\right) d t .
$$

(2) $\left(C_{\alpha, p, \infty}(E)\right)^{\frac{1}{p}} \leq P_{\alpha, p, \infty}(E)$.

(3) If $0<H^{n}(E), H^{n-1}(E)<\infty$, then

$$
P_{\alpha, p, \infty}(E)<\infty \Leftrightarrow \alpha \leq p^{-1},
$$

in other words,

$$
P_{\alpha, p, \infty}(E)=\infty \Leftrightarrow \alpha>p^{-1} .
$$

(4) Let $\mathbb{B}^{n}$ be the unit ball in $\mathbb{R}^{n}$. For any Euclidean ball $B\left(x_{0}, r_{0}\right)$ centered at $x_{0} \in \mathbb{R}^{n}$ and with radius $r_{0}>0$,

$$
C_{\alpha, p, \infty}\left(B\left(x_{0}, r_{0}\right)\right)=r_{0}^{n-\alpha p} C_{\alpha, p, \infty}\left(\mathbb{B}^{n}\right)
$$

$$
C_{\alpha, p, \infty}(E) \leq\left\{\begin{array}{l}
\left(\frac{\pi^{\frac{n-\alpha p}{2}}}{\Gamma\left(1+\frac{n-\alpha p}{2}\right)}\right)^{-1} C_{\alpha, p, \infty}\left(\mathbb{B}^{n}\right) H^{n-\alpha p}(E) \text { as } \quad p \in\left[1, \frac{n}{\alpha}\right) \\
0 \quad \text { as } \quad p \in\left[\frac{n}{\alpha}, \infty\right)
\end{array}\right.
$$

Proof. (1) Without loss of generality, we may assume that $f \in \Lambda_{\alpha}^{p, \infty} \cap C\left(\mathbb{R}^{n}\right)$ is nonnegative. Note that if

$$
\{f>t\}=\left\{x \in \mathbb{R}^{n}: f(x)>t\right\}
$$

(the upper $0<t$-level set of $f$ ) then $f / t>1$ in $\{f>t\}$. Therefore,

$$
C_{\alpha, p, \infty}(\{f>t\}) \leq\|f / t\|_{\dot{\Lambda}_{\alpha}^{p, \infty}}^{p}
$$

and the desired weak-type estimate follows:

$$
t^{p} C_{\alpha, p, \infty}(\{f>t\}) \leq\|f\|_{\dot{\Lambda}_{\alpha}^{p, \infty}}^{p} \quad \forall \quad t \in(0, \infty) .
$$

To verify the remaining inequality in (1) (viewed as a co-area inequality), we use (cf. [3])

$$
\left|\Delta_{h} f(x)\right|=\int_{0}^{\infty}\left|1_{\{f>t\}}(x+h)-1_{\{f>t\}}(x)\right| d t
$$

and Minkowski's inequality, to derive

$$
|h|^{-\alpha}\left\|\Delta_{h} f\right\|_{L^{p}} \leq \int_{0}^{\infty}|h|^{-\alpha}\left\|1_{\{f>t\}}(\cdot+h)-1_{\{f>t\}}(\cdot)\right\|_{L^{p}} d t \leq \int_{0}^{\infty}\left\|1_{\{f>t\}}\right\|_{\Lambda_{\alpha}^{p, \infty}} d t,
$$

as desired.

(2) For any $f \in \mathrm{A}_{\alpha, p, \infty}(E)$ with $\|f\|_{\Lambda_{\alpha}^{p, \infty}} \rightarrow\left\|1_{E}\right\|_{\dot{\Lambda}_{\alpha}^{p, \infty}}$ we use the monotonicity of $C_{\alpha, p, \infty}$ to get

$$
t\left(C_{\alpha, p, \infty}(E)\right)^{\frac{1}{p}} \leq\left(t^{p} C_{\alpha, p, \infty}\left(\left\{x \in \mathbb{R}^{n}: f(x)>t\right\}\right)\right)^{\frac{1}{p}} \leq\|f\|_{\dot{\Lambda}_{\alpha}^{p, \infty}} \forall t \in(0,1),
$$

and, therefore,

$$
\left(C_{\alpha, p, \infty}(E)\right)^{\frac{1}{p}} \leq P_{\alpha, p, \infty}(E) .
$$

(3) Suppose $0<H^{n}(E), H^{n-1}(E)<\infty$. If $P_{\alpha, p, \infty}(E)$ is finite, then

$$
\left\|1_{E}(x+h)-1_{E}(x)\right\|_{L^{p}}^{p} \leq\left(P_{\alpha, p, \infty}(E)\right)^{p}|h|^{p \alpha} \quad \forall \quad h \in \mathbb{R}^{n} .
$$


A straightforward computation gives

$\left\|1_{E}(x+h)-1_{E}(x)\right\|_{L^{p}}^{p}=|E|+|E-h|-2|(E-h) \cap E|=2(|E|-|(E-h) \cap E|)=\left\|1_{E}-1_{E-h}\right\|_{L^{1}}$.

Therefore, for each natural number $k$, we have

$$
\left\|1_{E}-1_{E-h}\right\|_{L^{1}} \leq \sum_{j=0}^{k-1}\left\|1_{E-\frac{j}{k} h}-1_{E-\frac{j+1}{k} h}\right\|_{L^{1}} \leq\left(P_{\alpha, p, \infty}(E)\right)^{p} k^{1-p \alpha}|h|^{p \alpha} .
$$

Now, if $\alpha>p^{-1}$, then letting $k \rightarrow \infty$ in the last estimation gives

$$
\left\|1_{E}-1_{E-h}\right\|_{L^{1}}=0 \quad \forall \quad h \in \mathbb{R}^{n},
$$

and hence

$$
\lim _{|h| \rightarrow \infty}\left\|1_{E}-1_{E-h}\right\|_{L^{1}}=0
$$

However, we have

$$
\lim _{|h| \rightarrow \infty}\left\|1_{E}-1_{E-h}\right\|_{L^{1}}=2|E| \in(0, \infty),
$$

thereby reaching a contradiction. Therefore, we must have $\alpha \leq p^{-1}$. Conversely, if $\alpha \leq p^{-1}$ holds, then an application of [26. Theorem 1] to the symmetric difference of $E$ and $E-h$ readily shows that

$$
2(|E|-|E \cap(E-h)|) \leq|h| H^{n-1}(E) \quad \forall \quad h \in \mathbb{R}^{n} .
$$

Therefore, by the classical isoperimetric inequality

$$
\left(\frac{H^{n}(E)}{\left(\frac{\pi^{\frac{n}{2}}}{\Gamma\left(1+\frac{n}{2}\right)}\right)}\right)^{\frac{1}{n}} \leq\left(\frac{H^{n-1}(E)}{\left(\frac{\pi^{\frac{n-1}{2}}}{\Gamma\left(1+\frac{n-1}{2}\right)}\right)}\right)^{\frac{1}{n-1}}
$$

and the hypothesis $p^{-1}-\alpha \geq 0$, we find

$$
\begin{aligned}
P_{\alpha, p, \infty}(E) & \leq \max \left\{\sup _{h \in \mathbb{B}^{n}}|h|^{p^{-1}-\alpha}\left(H^{n-1}(E)\right)^{\frac{1}{p}}, \sup _{h \in \mathbb{R}^{n} \backslash \mathbb{B}^{n}}|h|^{-\alpha}\left(2 H^{n}(E)\right)^{\frac{1}{p}}\right\} \\
& \leq\left(H^{n-1}(E)\right)^{\frac{1}{p}}+\left(2 H^{n}(E)\right)^{\frac{1}{p}} \\
& \leq\left(H^{n-1}(E)\right)^{\frac{1}{p}}+\left(2\left(\frac{\pi^{\frac{n}{2}}}{\Gamma\left(1+\frac{n}{2}\right)}\right)\left(\frac{H^{n-1}(E)}{\left(\frac{\pi^{\frac{n-1}{2}}}{\Gamma\left(1+\frac{n-1}{2}\right)}\right)}\right)^{\frac{n}{n-1}}\right)^{\frac{1}{p}} \\
& <\infty .
\end{aligned}
$$

(4) We notice that if $f \in \mathrm{A}_{\alpha, p, \infty}\left(B\left(x_{0}, r_{0}\right)\right)$ and $f_{r_{0}}(x)=f\left(r_{0} x\right)$ then

$$
\left\|f_{r_{0}}\right\|_{\dot{\Lambda}_{\alpha}^{p, \infty}}^{p}=r_{0}^{\alpha p-n}\|f\|_{\dot{\Lambda}_{\alpha}^{p, \infty}}^{p} .
$$

Therefore, by the definition of $C_{\alpha, p, \infty}$, we the desired formula follows.

(5) Let $p \in\left[1, \frac{n}{\alpha}\right.$ ). Using Propositions 2(2)-(3) \& 3(4) we see that if $E \subseteq \cup_{j=1}^{\infty} B\left(x_{j}, r_{j}\right)$ then

$$
C_{\alpha, p, \infty}(E) \leq \sum_{j=1}^{\infty} C_{\alpha, p, \infty}\left(B\left(x_{j}, r_{j}\right)\right)=\sum_{j=1}^{\infty} r_{j}^{n-\alpha p} C_{\alpha, p, \infty}\left(\mathbb{B}^{n}\right) .
$$


Therefore, by the definition of $H^{n-\alpha p}(E)$, we have

$$
C_{\alpha, p, \infty}(E) \leq\left(\frac{\pi^{\frac{n-\alpha p}{2}}}{\Gamma\left(1+\frac{n-\alpha p}{2}\right)}\right)^{-1} H^{n-\alpha p}(E) C_{\alpha, p, \infty}\left(\mathbb{B}^{n}\right) .
$$

If $p \geq \frac{n}{\alpha}$, then in view of Proposition 1 and the ball-decomposition of open sets in $\mathbb{R}^{n}$, it is enough to verify the result for balls, $B\left(x_{0}, R_{0}\right)$. We shall consider two cases: Suppose first that $p>\frac{n}{\alpha}$, then, as $R_{0} \rightarrow \infty$, we have

$$
C_{\alpha, p, \infty}\left(B\left(x_{0}, R_{0}\right)\right)=R_{0}^{n-\alpha p} C_{\alpha, p, \infty}\left(\mathbb{B}^{n}\right) \rightarrow 0 .
$$

Since $C_{\alpha, p, \infty}$ is monotone, we see that

$$
C_{\alpha, p, \infty}\left(B\left(x_{0}, r_{0}\right)\right) \leq C_{\alpha, p, \infty}\left(B\left(x_{0}, R_{0}\right) \rightarrow 0 \text { as } R_{0} \rightarrow \infty\right.
$$

whence

$$
C_{\alpha, p, \infty}\left(B\left(x_{0}, r_{0}\right)\right)=0
$$

Suppose now that $p=\frac{n}{\alpha}$. We have,

$$
C_{\alpha, p, \infty}\left(B\left(x_{0}, R_{0}\right)\right)=C_{\alpha, p, \infty}\left(\mathbb{B}^{n}\right) \forall R_{0}>0 .
$$

By Proposition 2(5) we have

$$
\lim _{R_{0} \rightarrow 0} C_{\alpha, p, \infty}\left(B\left(x_{0}, R_{0}\right)\right)=0
$$

whence

$$
C_{\alpha, p, \infty}\left(B\left(x_{0}, r_{0}\right)\right)=C_{\alpha, p, \infty}\left(\mathbb{B}^{n}\right)=0 \quad \forall r_{0}>0
$$

Corollary 4. Let $K$ be a compact subset of $\mathbb{R}^{n}$ and let $\partial K$ be its boundary. Let $\mathrm{O}(K)$ stand for the class of all open sets $O \subset \mathbb{R}^{n}$ with $O \supset K$.

(1) If $(\alpha, p) \in(0,1) \times\left[1, \frac{n}{\alpha}\right)$, then

$$
C_{\alpha, p, \infty}(K)=C_{\alpha, p, \infty}(\partial K)
$$

(2) If $(\alpha, p) \in(0,1) \times\left[1, \frac{1}{\alpha}\right)$, then

$$
C_{\alpha, p, \infty}(K) \leq \inf _{O \in \mathrm{O}(K)}\left(P_{\alpha, p, \infty}(O)\right)^{p}
$$

with equality when $(\alpha, p) \in(0,1) \times\{1\}$, i.e.,

$$
C_{\alpha, 1, \infty}(K)=\inf _{O \in \mathrm{O}(K)} P_{\alpha, 1, \infty}(O) .
$$

Proof. (1) By Propositions 2(2)\&3(5), it is enough to prove

$$
C_{\alpha, p, \infty}(K) \leq C_{\alpha, p, \infty}(\partial K)
$$

Given $f \in \mathrm{A}_{\alpha, p, \infty}(\partial K)$ let us define

$$
g=\left\{\begin{array}{l}
\max \{f, 1\} \quad \text { on } \quad K \\
f \quad \text { on } \quad \mathbb{R}^{n} \backslash K
\end{array}\right.
$$

Then,

$$
g \in \mathrm{A}_{\alpha, p, \infty}(K) \&\|g\|_{\dot{\Lambda}_{\alpha}^{p, \infty}}^{p} \leq\|f\|_{\dot{\Lambda}_{\alpha}^{p, \infty}}^{p}
$$


Combining this fact with the definition of $C_{\alpha, p, \infty}(K)$, readily yields

$$
C_{p, \alpha, \infty}(K) \leq\|f\|_{\dot{\Lambda}_{\alpha}^{p, \infty}}^{p},
$$

and the result follows.

(2) The desired inequality follows from Propositions 2(2) \& 3(2). Now, suppose that $p=1$ and $f \in \mathrm{A}_{\alpha, 1, \infty}(K)$. Note that, if $t \in(0,1)$, then the upper-level set $\left\{x \in \mathbb{R}^{n}: f(x)>t\right\}$, is an open set containing $K$. Given $h \in \mathbb{R}^{n}$, and $\epsilon>0$, there exists $\tilde{O} \in \mathrm{O}(K)$ such that

$$
\inf _{O \in \mathrm{O}(K)}\left\|1_{O}(\cdot+h)-1_{O}(\cdot)\right\|_{L^{1}} \geq\left\|1_{\tilde{O}}(\cdot+h)-1_{\tilde{O}}(\cdot)\right\|_{L^{1}}-\epsilon .
$$

Therefore, by Fubini's theorem, it follows that

$$
\begin{aligned}
\|f\|_{\Lambda_{\alpha}^{1, \infty}} & =\sup _{h \in \mathbb{R}^{n}}|h|^{-\alpha}\left\|\Delta_{h} f\right\|_{L^{1}} \\
& =\sup _{h \in \mathbb{R}^{n}} \int_{0}^{\infty}|h|^{-\alpha}\left\|1_{\{f>t\}}(\cdot+h)-1_{\{f>t\}}(\cdot)\right\|_{L^{1}} d t \\
& \geq \sup _{h \in \mathbb{R}^{n}} \int_{0}^{1}|h|^{-\alpha}\left\|1_{\{f>t\}}(\cdot+h)-1_{\{f>t\}}(\cdot)\right\|_{L^{1}} d t \\
& \geq \sup _{h \in \mathbb{R}^{n}} \int_{0}^{1}|h|^{-\alpha} \inf _{O \in \mathrm{O}(K)}\left\|1_{O}(\cdot+h)-1_{O}(\cdot)\right\|_{L^{1}} d t \\
& =\sup _{h \in \mathbb{R}^{n}}|h|^{-\alpha} \inf _{O \in \mathrm{O}(K)}\left\|1_{O}(\cdot+h)-1_{O}(\cdot)\right\|_{L^{1}} \\
& \geq \sup _{h \in \mathbb{R}^{n}}|h|^{-\alpha}\left(\left\|1_{\tilde{O}}(\cdot+h)-1_{\tilde{O}}(\cdot)\right\|_{L^{1}}-\epsilon\right) \\
& \geq \sup _{h \in \mathbb{R}^{n}}|h|^{-\alpha}\left\|1_{\tilde{O}}(\cdot+h)-1_{\tilde{O}}(\cdot)\right\|_{L^{1}}-\epsilon \inf _{h \in \mathbb{R}^{n}}|h|^{-\alpha} \\
& =P_{\alpha, 1, \infty}(\tilde{O}) \\
& \geq \inf _{O \in \mathrm{O}(K)} P_{\alpha, 1, \infty}(O) .
\end{aligned}
$$

As a consequence, we find

$$
C_{\alpha, 1, \infty}(K)=\inf _{O \in \mathrm{O}(K)} P_{\alpha, 1, \infty}(O)
$$

\section{3. $\infty$-BESOV RESTRICTIONS, EXTENSIONS AND MULTIPLIERS}

3.1. $\infty$-Besov restrictions. Proposition 3 tells us that $C_{\alpha, p, \infty}$ is interesting only when $1 \leq$ $p<\frac{n}{\alpha}$. Moreover, according to Proposition 2 this capacity is an outer measure. Therefore, it is a good fit for the so-called Lebesgue theory for outer measures developed recently in [9]. We can thus try to measure the trace/restriction of a $\Lambda_{\alpha}^{p, \infty}$-function on a given compact exceptional set via looking for an outer measure $\mu$ concentrated on this compact set such that $\Lambda_{\alpha}^{p, \infty}$ embeds continuously into a weak $\mu$-based Lorentz space. More precisely, we have the following trace/restriction result.

Proposition 5. Let $(\alpha, p, q) \in(0,1) \times\left[1, \frac{n}{\alpha}\right) \times[1, \infty)$, and let $\mu$ be a nonnegative outer measure on $\mathbb{R}^{n}$. Consider the following statements:

(1) $\sup _{t \in(0, \infty)}\left(t^{q} \mu\left(\left\{x \in \mathbb{R}^{n}:|f(x)|>t\right\}\right)\right)^{\frac{1}{q}} \lesssim\|f\|_{\Lambda_{\alpha}^{p, \infty}} \forall f \in \Lambda_{\alpha}^{p, \infty} \cap C\left(\mathbb{R}^{n}\right)$. 
(2) $\mu(E) \lesssim\left(C_{\alpha, p, \infty}(E)\right)^{\frac{q}{p}} \forall$ Borel set $E \subset \mathbb{R}^{n}$.

(3) $\sup _{(t, x, r) \in(0, \infty) \times \mathbb{R}^{n} \times(0, \infty)}\left(t^{q} \mu(\{y \in B(x, r):|f(y)|>t\})\right)^{\frac{1}{q}} \lesssim\|f\|_{\Lambda_{\alpha}^{p, \infty}} \forall f \in \Lambda_{\alpha}^{p, \infty} \cap C\left(\mathbb{R}^{n}\right)$.

(4) $\mu(B(x, r)) \lesssim r^{\frac{q(n-\alpha p)}{p}} \forall$ Euclidean ball $B(x, r) \subset \mathbb{R}^{n}$.

Then, the following equivalences hold: (1) $\Leftrightarrow$ (2) and (3) $\Leftrightarrow$ (4).

Proof. (1) $\Leftrightarrow$ (2) Suppose that (1) holds. Let $f \in \mathrm{A}_{\alpha, p, \infty}(E)$, and let $E \subset \mathbb{R}^{n}$ be a Borel set. Then,

$$
t^{q} \mu(E) \leq t^{q} \mu\left(\left\{x \in \mathbb{R}^{n}:|f(x)|>t\right\}\right) \lesssim\|f\|_{\dot{\Lambda}_{\alpha}^{p, \infty}}^{q} \forall t \in(0,1) .
$$

Letting $t$ tend to 1 , and using the definition of $C_{\alpha, p, \infty}(E)$ readily yields (2). Conversely, if (2) holds true, then an application of Proposition 3(1) gives

$$
t^{q} \mu\left(\left\{x \in \mathbb{R}^{n}:|f(x)|>t\right\}\right) \lesssim\left(t^{p} C_{\alpha, p, \infty}\left(\left\{x \in \mathbb{R}^{n}:|f(x)|>t\right\}\right)\right)^{\frac{q}{p}} \lesssim\|f\|_{\dot{\Lambda}_{\alpha}^{p, \infty}}^{q},
$$

whence (1).

(3) $\Leftrightarrow$ (4) Suppose that (3) is valid, let $f \in \mathrm{A}_{\alpha, p, \infty}(B(x, r))$, where $B(x, r)$ is an Euclidean ball. Then,

$$
t^{q} \mu(B(x, r)) \leq t^{q} \mu(\{y \in B(x, r):|f(y)|>t\}) \lesssim\|f\|_{\dot{\Lambda}_{\alpha}^{p, \infty}}^{q} \forall t \in(0,1) .
$$

Letting $t \rightarrow 1$, and using the definition of $C_{\alpha, p, \infty}(B(x, r))$, as well as Proposition 3(4), yields

$$
\mu(B(x, r)) \lesssim\left(C_{\alpha, p, \infty}(B(x, r))\right)^{\frac{q}{p}} \approx r^{\frac{q(n-\alpha p)}{p}},
$$

whence (4). Conversely, suppose that (4) is true. Applying Proposition 3(1) we find that

$$
t^{q} \mu(\{y \in B(x, r):|f(x)|>t\}) \lesssim\left(t^{p} C_{\alpha, p, \infty}(\{y \in B(x, r):|f(y)|>t\})\right)^{\frac{q}{p}} \lesssim\|f\|_{\dot{\Lambda}_{\alpha}^{p, \infty}}^{q}
$$

holds for any Euclidean ball $B(x, r) \subset \mathbb{R}^{n}$, whence (3) holds.

Remark 4. Three comments are in order.

(i) If $\|f\|_{L_{\mu}^{q, \infty}}=\sup _{t \in(0, \infty)}\left(t^{q} \mu\left(\left\{x \in \mathbb{R}^{n}:|f(x)|>t\right\}\right)\right)^{\frac{1}{q}}$, then Lyapunov's inequality (cf. [6, Proposition 5.3]) is:

$$
\|f\|_{L_{\mu}^{q_{\mu}, \infty}} \leq\|f\|_{L_{\mu}^{q_{0}, \infty}}^{1-\theta}\|f\|_{L_{\mu}^{q_{1}, \infty}}^{\theta} \quad \forall \quad \frac{1}{q}=\frac{1-\theta}{q_{0}}+\frac{\theta}{q_{1}} \&\left(\theta, q_{0}, q_{1}\right) \in(0,1) \times[1, \infty) \times[1, \infty) .
$$

Similarly, one has:

$$
\|f\|_{\dot{\Lambda}_{\alpha}^{p, \infty}} \leq\|f\|_{\dot{\Lambda}_{\alpha}^{p_{0}, \infty}}^{1-\theta}\|f\|_{\dot{\Lambda}_{\alpha}^{p_{1}, \infty}}^{\theta} \quad \forall \quad \frac{1}{p}=\frac{1-\theta}{p_{0}}+\frac{\theta}{p_{1}} \&\left(\theta, p_{0}, p_{1}\right) \in(0,1) \times[1, \infty) \times[1, \infty) .
$$

(ii) If we let $\mu$ be the n-dimensional Lebesgue measure in Proposition 51) and we have $n-\alpha p>0$, then the limiting case of [19, Theorem 2.8] or [27, Corollary 3.3] ensures

$$
\|f\|_{L_{\mu}^{p-\alpha n}, \infty} \leq 2^{\frac{1}{p}}\left(\frac{p n}{n-\alpha p}\right)\|f\|_{\Lambda_{\alpha}^{p, \infty}} \quad \forall \quad f \in \Lambda_{\alpha}^{p, \infty} \cap C\left(\mathbb{R}^{n}\right) .
$$


Therefore, Proposition 5 (1) is valid for $q=\frac{p n}{n-\alpha p}$ and the $n$-dimensional Lebesgue measure. Consequently, one has the following isocapacitary - isoperimetric inequality

$$
|E|^{\frac{n-\alpha p}{p n}} \leq 2^{\frac{1}{p}}\left(\frac{p n}{n-\alpha p}\right)\left(C_{\alpha, p, \infty}(E)\right)^{\frac{1}{p}} \leq 2^{\frac{1}{p}}\left(\frac{p n}{n-\alpha p}\right) P_{\alpha, p, \infty}(E) \quad \forall \quad \text { Borel set } \quad E \subset \mathbb{R}^{n} .
$$

We should also remark that, due to Corollary 4 the important case here corresponds to $p \in\left[1, \alpha^{-1}\right]$.

(iii) Proposition 5(2) implies Proposition 5(4), but the converse does not necessarily hold.

Corollary 6. Let $(\alpha, p, q) \in(0,1) \times\left[1, \frac{n}{\alpha}\right) \times[1, \infty)$, let $\mu$ be a nonnegative outer measure on $\mathbb{R}^{n}$, and let $\phi: \mathbb{R}^{n} \rightarrow \mathbb{R}^{n}$ be a Borel map. Referring to the numbered statements below the following equivalences hold true: (1) $\Leftrightarrow$ (2) and (3) $\Leftrightarrow$ (4).

(1) $\|f \circ \phi\|_{L_{\mu}^{q, \infty}} \lesssim\|f\|_{\dot{\Lambda}_{\alpha}^{p, \infty}} \forall f \in \Lambda_{\alpha}^{p, \infty} \cap C\left(\mathbb{R}^{n}\right)$.

(2) $\mu\left(\phi^{-1}(E)\right) \lesssim\left(C_{\alpha, p, \infty}(E)\right)^{\frac{q}{p}} \forall$ Borel set $E \subset \mathbb{R}^{n}$.

(3) $\sup _{(t, x, r) \in(0, \infty) \times \mathbb{R}^{n} \times(0, \infty)}\left(t^{q} \mu\left(\left\{y \in \phi^{-1}(B(x, r)):|f \circ \phi(y)|>t\right\}\right)\right)^{\frac{1}{q}} \lesssim\|f\|_{\Lambda_{\alpha}^{p, \infty}} \forall f \in$ $\Lambda_{\alpha}^{p, \infty} \cap C\left(\mathbb{R}^{n}\right)$.

(4) $\mu\left(\phi^{-1}(B(x, r))\right) \lesssim r^{\frac{q(n-\alpha p)}{p}} \forall$ Euclidean ball $B(x, r) \subset \mathbb{R}^{n}$.

Proof. Clearly, the above conclusions follow by means of applying Proposition 5 to the pushforward outer measure $\phi_{*} \mu$ defined by:

$$
\phi_{*} \mu(E)=\mu\left(\phi^{-1}(E)\right) \quad \forall \quad \text { Borel set } E \subset \mathbb{R}^{n} .
$$

3.2. $\infty$-Besov extensions. Lifting an arbitrary $\Lambda_{\alpha}^{p, \infty}$-function to the upper-half space $\mathbb{R}_{+}^{1+n}=$ $(0, \infty) \times \mathbb{R}^{n}$ via the heat equation, we obtain the Carleson imbedding for $\dot{\Lambda}_{\alpha}^{p, \infty}$ via the heat equation (which can be also generalized to the fractional case; see [36]).

Proposition 7. Let $(\alpha, p, q) \in(0,1) \times\left(1, \frac{n}{\alpha}\right) \times[1, \infty)$ and $v$ be a nonnegative outer measure on $\mathbb{R}_{+}^{1+n}$. Let

$$
w(t, x)=(4 \pi t)^{-\frac{n}{2}} \int_{\mathbb{R}^{n}} \exp \left(-\frac{|x-y|^{2}}{4 t}\right) f(y) d y,
$$

be the solution to the heat equation

$$
\left\{\begin{array}{l}
\left(\partial_{t}-\Delta_{x}\right) w(t, x)=0 \quad \forall \quad(t, x) \in \mathbb{R}_{+}^{1+n} \\
w(0, x)=f(x) \quad x \in \mathbb{R}^{n}
\end{array}\right.
$$

For an open set $O \subset \mathbb{R}^{n}$, let $T(O)=\left\{(t, x) \in \mathbb{R}_{+}^{1+n}: B(x, t) \subseteq O\right\}$, be the tent with base $O \subset \mathbb{R}^{n}$. Then,

$$
\begin{aligned}
& \sup _{\lambda \in(0, \infty)}\left(\lambda^{q} v\left(\left\{(t, x) \in \mathbb{R}_{+}^{1+n}:\left|w\left(t^{2}, x\right)\right|>\lambda\right\}\right)\right)^{\frac{1}{q}} \lesssim\|f\|_{\Lambda_{\alpha}^{p, \infty}} \forall f \in \Lambda_{\alpha}^{p, \infty} \cap C\left(\mathbb{R}^{n}\right) \\
& \Longleftrightarrow v(T(O)) \lesssim\left(C_{\alpha, p, \infty}(O)\right)^{\frac{q}{p}} \forall \text { open set } O \subset \mathbb{R}^{n} .
\end{aligned}
$$


Proof. Suppose that

$$
\sup _{\lambda \in(0, \infty)}\left(\lambda^{q} v\left(\left\{(t, x) \in \mathbb{R}_{+}^{1+n}:\left|w\left(t^{2}, x\right)\right|>\lambda\right\}\right)\right)^{\frac{1}{q}} \lesssim\|f\|_{\Lambda_{\alpha}^{p, \infty}} \forall f \in \Lambda_{\alpha}^{p, \infty} \cap C\left(\mathbb{R}^{n}\right)
$$

holds; then, for any given open set $O \subset \mathbb{R}^{n}$, we can pick $f \in \mathrm{A}_{\alpha, p, \infty}(O)$ and find a dimensional constant $c>0$ such that

$$
w\left(t^{2}, x\right) \geq(4 \pi t)^{-\frac{n}{2}} \int_{B(x, t)} \exp \left(-\frac{|x-y|^{2}}{4 t}\right) f(y) d y \geq c \quad \forall \quad(t, x) \in T(O) .
$$

Then, by the definition of $C_{\alpha, p, \infty}(O)$, we get

$$
v(T(O)) \lesssim\left(C_{\alpha, p, \infty}(O)\right)^{\frac{q}{p}} .
$$

On the other hand, suppose that the last estimate holds true for any open set $O \subset \mathbb{R}^{n}$. Then, if $f \in \Lambda_{\alpha}^{p, \infty} \cap C\left(\mathbb{R}^{n}\right)$, we let

$$
\mathcal{M}_{N} f(x)=\sup _{|y-x|<t}\left|w\left(t^{2}, y\right)\right|
$$

i.e. the non-tangential maximal function of $w\left(t^{2}, y\right)$. Since $\mathcal{M}_{N} f$ is lower semi-continuous, the level sets $\left\{x \in \mathbb{R}^{n}: \mathcal{M}_{N} f(x)>\lambda\right\}$ are open for all $\lambda>0$. Moreover, (cf. [15])

$$
\left|w\left(t^{2}, x-y\right)\right| \lesssim\left(1+\left(|y| t^{-1}\right)^{2}\right) \mathcal{M} f(x),
$$

where $\mathcal{M} f$ is the standard Hardy-Littlewood maximal function of $f$ :

$$
\mathcal{M} f(x)=\sup _{r>0}|B(x, r)|^{-1} \int_{B(x, r)}|f(y)|, d y .
$$

Therefore, there exists a constant $c_{0}>0$ such that $\mathcal{M}_{N} f \leq c_{0} \mathcal{M} f$. Consequently,

$$
\begin{aligned}
v\left(\left\{(t, x) \in \mathbb{R}_{+}^{1+n}:\left|w\left(t^{2}, x\right)\right|>\lambda\right\}\right) & \leq v\left(T\left(\left\{x \in \mathbb{R}^{n}: \mathcal{M}_{N} f(x)>\lambda\right\}\right)\right) \\
& \leq v\left(T\left(\left\{x \in \mathbb{R}^{n}: \mathcal{M} f(x)>c_{0} \lambda\right\}\right)\right) \\
& \lesssim\left(C_{\alpha, p, \infty}\left(\left\{x \in \mathbb{R}^{n}: \mathcal{M} f(x)>c_{0} \lambda\right\}\right)\right)^{\frac{q}{p}} .
\end{aligned}
$$

Since $\mathcal{M}$ is bounded on $L^{p}$ we see that (cf. [31, p.3247])

$$
\left\|\Delta_{h}(\mathcal{M} f)\right\|_{L^{p}} \lesssim\left\|\mathcal{M}\left(\Delta_{h} f\right)\right\|_{L^{p}} \lesssim\left\|\Delta_{h} f\right\|_{L^{p}} \quad \forall \quad(p, h) \in(1, \infty) \times \mathbb{R}^{n} .
$$

Using Proposition 2(1), we get

$$
\left(\lambda^{p} C_{\alpha, p, \infty}\left(\left\{x \in \mathbb{R}^{n}: \mathcal{M} f(x)>c_{0} \lambda\right\}\right)\right)^{\frac{q}{p}} \lesssim\|\mathcal{M} f\|_{\dot{\Lambda}_{\alpha}^{p, \infty}}^{q} \lesssim\|f\|_{\dot{\Lambda}_{\alpha}^{p, \infty}}^{q},
$$

whence

$$
\lambda^{q} v\left(\left\{(t, x) \in \mathbb{R}_{+}^{1+n}:\left|w\left(t^{2}, x\right)\right|>\lambda\right\}\right) \lesssim\|f\|_{\dot{\Lambda}_{\alpha}^{p, \infty}}^{q}
$$


3.3. $\infty$-Besov point-wise multipliers. In view of Proposition 5 , we can naturally deal with the following multiplication problem for $\dot{\Lambda}_{\alpha}^{p, \infty}$.

Proposition 8. Let $(\alpha, p, q) \in(0,1) \times\left[1, \frac{n}{\alpha}\right) \times[1, \infty)$, let $\mu$ be a nonnegative outer measure on $\mathbb{R}^{n}$, and let $\mathrm{m}: \mathbb{R}^{n} \rightarrow \mathbb{R}$ be a Borel function. Then, one has the following implications:

$$
\begin{aligned}
& \mathrm{m} \in L_{\mu}^{\infty} \& \mu(E) \lesssim\left(C_{\alpha, p, \infty}(E)\right)^{\frac{q}{p}} \forall \text { Borel set } E \subset \mathbb{R}^{n} \\
& \Longrightarrow\|\mathrm{m} f\|_{L_{\mu}^{q, \infty}} \lesssim\|f\|_{\Lambda_{\alpha}^{p, \infty}} \forall f \in \Lambda_{\alpha}^{p, \infty} \cap C\left(\mathbb{R}^{n}\right) \\
& \Longrightarrow \sup _{t \in(0, \infty)} t^{q} \mu(\{x \in E:|\mathrm{m}(x)|>t\}) \lesssim\left(C_{\alpha, p, \infty}(E)\right)^{\frac{q}{p}} \forall \text { Borel set } E \subset \mathbb{R}^{n} .
\end{aligned}
$$

$$
\begin{aligned}
& \mathrm{m} \in L_{\mu}^{\infty} \& \mu(B(x, r)) \lesssim r^{\frac{q(n-\alpha p)}{p}} \forall \text { Euclidean ball } B(x, r) \subset \mathbb{R}^{n} \\
& \Longrightarrow \sup _{(t, x, r) \in(0, \infty) \times \mathbb{R}^{n} \times(0, \infty)}\left(t^{q} \mu(\{y \in B(x, r):|\mathrm{m}(y) f(y)|>t\})\right)^{\frac{1}{q}} \lesssim\|f\|_{\Lambda_{\alpha}^{p, \infty}} \forall f \in \Lambda_{\alpha}^{p, \infty} \cap C\left(\mathbb{R}^{n}\right) \\
& \Longrightarrow \sup _{t \in(0, \infty)} t^{q} \mu(\{y \in B(x, r):|\mathrm{m}(y)|>t\}) \lesssim r^{\frac{q(n-\alpha p)}{p}} \forall \text { Euclidean ball } B(x, r) \subset \mathbb{R}^{n} .
\end{aligned}
$$

Proof. It is enough to check Proposition 8 (1). Suppose that

$$
\mathrm{m} \in L_{\mu}^{\infty} \& \mu(E) \lesssim\left(C_{\alpha, p, \infty}(E)\right)^{\frac{q}{p}} \forall \text { Borel set } E \subset \mathbb{R}^{n} .
$$

Without loss of generality we may assume that $\|\mathrm{m}\|_{L_{\mu}^{\infty}}>0$. Note that if $f \in \Lambda_{\alpha}^{p, \infty} \cap C\left(\mathbb{R}^{n}\right)$, then

$$
t<|\mathrm{m}(x) f(x)| \leq\|\mathrm{m}\|_{L_{\mu}^{\infty}}|f(x)| \Rightarrow t|| \mathrm{m} \|_{L_{\mu}^{\infty}}^{-1}<|f(x)| .
$$

Therefore, an application of Proposition $5(1) \Leftrightarrow(2)$, yields

$$
\|\mathrm{m}\|_{L_{\mu}^{\infty}}^{q}\|f\|_{\Lambda_{\alpha}^{p, \infty}}^{q} \gtrsim\|\mathrm{m}\|_{L_{\mu}^{\infty}}^{q}\left(\frac{t}{\|\mathrm{~m}\|_{L_{\mu}^{\infty}}}\right)^{q} \mu\left(\left\{x \in \mathbb{R}^{n}:|f(x)|>\frac{t}{\|\mathrm{~m}\|_{L_{\mu}^{\infty}}}\right\}\right) \geq\|\mathrm{m} f\|_{L_{\mu}^{q, \infty}}^{q} .
$$

Next, we assume

$$
\|\mathrm{m} f\|_{L_{\mu}^{q, \infty}} \lesssim\|f\|_{\Lambda_{\alpha}^{p, \infty}} \forall f \in \Lambda_{\alpha}^{p, \infty} \cap C\left(\mathbb{R}^{n}\right),
$$

Let $E$ by a Borel set in $\mathbb{R}^{n}$, and let $g \in \mathrm{A}_{p, \infty, \alpha}(E)$. Then,

$$
\{x \in E:|\operatorname{m}(x) g(x)|>t\} \supseteq\{x \in E:|\mathrm{m}(x)|>t\} \quad \forall \quad t \in(0, \infty) .
$$

Whence,

$$
\begin{aligned}
\|g\|_{\hat{\Lambda}_{\alpha}^{p, \infty}}^{q} & \gtrsim\|\mathrm{m} g\|_{L_{\mu}^{q, \infty}}^{q} \\
& \gtrsim \sup _{t \in(0, \infty)} t^{q} \mu(\{x \in E:|\mathrm{m}(x) g(x)|>t\}) \\
& \gtrsim \sup _{t \in(0, \infty)} t^{q} \mu(\{x \in E:|\operatorname{m}(x)|>t\}) .
\end{aligned}
$$

Taking the infimum over all such $g$ finally yields

$$
\left(C_{\alpha, p, \infty}(E)\right)^{\frac{q}{p}} \gtrsim \sup _{t \in(0, \infty)} t^{q} \mu(\{x \in E:|\mathrm{m}(x)|>t\}) .
$$


Remark 5. We ask wether the following implications

$$
\begin{aligned}
& \sup _{t \in(0, \infty)} t^{q} \mu(\{x \in E:|\mathrm{m}(x)|>t\}) \lesssim\|\mathrm{m}\|_{L_{\mu}^{\infty}}^{q}\left(C_{\alpha, p, \infty}(E)\right)^{\frac{q}{p}} \forall \text { Borel set } E \subset \mathbb{R}^{n} \\
& \Longrightarrow \mathrm{m} \in L_{\mu}^{\infty} \& \mu(E) \lesssim\left(C_{\alpha, p, \infty}(E)\right)^{\frac{q}{p}} \forall \text { Borel set } E \subset \mathbb{R}^{n}
\end{aligned}
$$

and

$$
\begin{aligned}
& \sup _{t \in(0, \infty)} t^{q} \mu(\{y \in B(x, r):|\mathrm{m}(y)|>t\}) \lesssim r^{\frac{q(n-\alpha p)}{p}} \forall \text { Euclidean ball } B(x, r) \subset \mathbb{R}^{n} \\
& \Longrightarrow \mathrm{m} \in L_{\mu}^{\infty} \& \mu(B(x, r)) \lesssim r^{\frac{q(n-\alpha p)}{p}} \forall \text { Euclidean ball } B(x, r) \subset \mathbb{R}^{n}
\end{aligned}
$$

are true or not? Obviously, if the essential lower bound of the $L_{\mu}^{\infty}$-function $\mathrm{m}$ is positive, then the answer to the above questions is affirmative.

Moreover, if $\mu$ is a nonnegative Radon measure, then [6, Proposition 5.1] implies

$$
\begin{aligned}
& \sup _{t \in(0, \infty)} t^{q} \mu(\{x \in E:|\mathrm{m}(x)|>t\}) \\
& \leq \sup _{0<\mu(E)<\infty} \mu(E)\left(\int_{E}|\mathrm{~m}|^{r} \frac{d \mu}{\mu(E)}\right)^{\frac{q}{r}} \\
& \leq\left(\frac{q}{q-r}\right)^{\frac{q}{r}} \sup _{t \in(0, \infty)} t^{q} \mu(\{x \in E:|\mathrm{m}(x)|>t\}) \quad \forall \quad r \in(0, q) .
\end{aligned}
$$

Letting $r \rightarrow 0$, and using [10, Exercise 6.117 (b)], we get

$$
\begin{aligned}
& \sup _{t \in(0, \infty)} t^{q} \mu(\{x \in E:|\mathrm{m}(x)|>t\}) \\
& \leq \sup _{0<\mu(E)<\infty} \mu(E) \exp \left(\int_{E} \log |\mathrm{m}|^{q} \frac{d \mu}{\mu(E)}\right) \\
& \leq e^{1 / p} \sup _{t \in(0, \infty)} t^{q} \mu(\{x \in E:|\mathrm{m}(x)|>t\}) .
\end{aligned}
$$

Thus,

$$
\begin{aligned}
& \sup _{t \in(0, \infty)} t^{q} \mu(\{x \in E:|\mathrm{m}(x)|>t\}) \lesssim\|\mathrm{m}\|_{L_{\mu}^{\infty}}^{q}\left(C_{\alpha, p, \infty}(E)\right)^{\frac{q}{p}} \forall \text { Borel set } E \subset \mathbb{R}^{n} \\
& \Longrightarrow \sup _{0<\mu(E)<\infty} \mu(E) \exp \left(\int_{E} \log |\mathrm{m}|^{q} \frac{d \mu}{\mu(E)}\right) \lesssim\left(C_{\alpha, p, \infty}(E)\right)^{\frac{q}{p}}
\end{aligned}
$$

Similarly, one has

$$
\begin{aligned}
& \sup _{t \in(0, \infty)} t^{q} \mu(\{y \in B(x, r):|\mathrm{m}(y)|>t\}) \lesssim r^{\frac{q(n-\alpha p)}{p}} \forall \text { Euclidean ball } B(x, r) \subset \mathbb{R}^{n} \\
& \Longrightarrow \sup _{(x, r) \in \mathbb{R}^{n} \times(0, \infty)} \mu(B(x, r)) \exp \left(\int_{B(x, r)} \log |\mathrm{m}|^{q} \frac{d \mu}{\mu(B(x, r))}\right) \lesssim r^{\frac{q(n-\alpha p)}{p}} .
\end{aligned}
$$




\section{REFERENCES}

[1] D. R. Adams, Besov capacity redux. J. Math. Sci. 162 (2009), 307-318.

[2] D. R. Adams and J. Xiao, Strong type estimates for homogeneous Besov capacities. Math. Ann. 325 (2003), 695-709.

[3] L. Ambrosio, G. De Philippis and L. Martinazzi. Gamma-convergence of nonlocal perimeter functionals. Manuscripta Math. 134 (2011), 377-403.

[4] J. Bourgain, H. Brézis and P. Mironescu, Limiting embedding theorems for $W^{s, p}$ when $s \rightarrow 1$ and applications. J. Anal. Math. 87 (2002), 77-101.

[5] J. Bourgain, H. Brézis and P. Mironescu, Another look at Sobolev spaces. In: J.L. Menaldi, E. Rofman, A. Sulem (Eds.), Optimal Control and Partial Differential Equations, a volume in honor of A. Bensoussan's 60th birthday, IOS Press, 2001, pp. 439-455.

[6] R. E. Castillo, F. A. V. Narvaez and J. C. R. Fernández, Multiplication and composition operators on weak $L_{p}$ spaces. Bull. Malays. Math. Sci. Soc. DOI 10.1007/s40840-014-0081-1.

[7] A. Cohen, W. Dahmen, I. Daubechies and R. DeVore, Harmonic analysis of the space BV. Rev. Mat. Iberoamericana 19 (2003), 235-263.

[8] S. Costea, Strong $A_{\infty}$-weights and scaling invariant Besov capacities. Rev. Mat. Iberoamericana 23 (2007), 1067-1114.

[9] Y. Do and C. Thiele, $L^{p}$ theory for outer measures and two themes of Lennart Carleson united. Bull. Amer. Math. Soc. 52 (2015), 249-296.

[10] C. George, Exercises in Integration, Springer-Verlag, New York Inc. 1984.

[11] M. Giaquinta, Multiple Integrals in the Calculus of Variations and Nonlinear Elliptic Systems. Ann. Math. Studies 105, Princeton University Press, Princeton, N.J., 1983.

[12] H. Hakkarainen and J. Kinnunen, The BV-capacity in metric spaces. Manuscripta Math. 132(2010)51-73.

[13] J. Heinonen, T. Kilpeläinen, and O. Martio, Nonlinear Potential Theory of Degenerate Elliptic Equations, Oxford University Press, 1993.

[14] F. John and L. Nirenberg, On functions of bounded mean oscillation. Comm. Pure Appl. Math. 14 (1961), 415-426.

[15] R. Johnson, Application of Carleson measures to partial differential equations and Fourier multiplier problems, in: Proc. Conf. on Harmonic Anal., Cortona, in: Lecture Notes in Math., vol 992, Springer, 1983, pp. 16-72.

[16] G. E. Karadzhov, M. Milman and J. Xiao, Limits of higher-order Besov spaces and sharp reiteration theorems. J. Funct. Anal. 221 (2005), 323-339.

[17] J. Kinnunen and O. Martio, The Sobolev capacity on metric spaces. Ann. Acad. Sci. Fenn. Math. 21 (1996), 367-382.

[18] J. Kinnunen and O. Martio, Choquet property for the Sobolev capacity in metric spaces. Proceedings on Analysis and Geometry (Russian) (Novosibirsk Akademgorodok, 1999), 285-290, Izdat. Ross. Akad. Nauk Sib. Otd. Inst. Mat., Novosibirsk, 2000.

[19] V.I. Kolyada and A.K. Lerner, On limiting embeddings of Besov spaces. Studia Math. 171 (2005), 1-13.

[20] H. Kozono and Y. Taniuchi, Bilinear estimates in BMO and the Navier-Stokes equations. Math. Z. 235(2000), 173-194.

[21] A. Kufner, O. John and S. Fučik. Function Spaces, Noordhoff International Publishing, 1977.

[22] V. Maz'ya and T. Shaposhnikova, On the Bourgain, Brezis, and Mironescu theorem concerning limiting embeddings of fractional Sobolev spaces. J. Funct. Anal. 195 (2002), 230-238.

[23] V. Maz'ya and T. Shaposhnikova, Erratum to "On J. Bourgain, H. Brézis and P. Mironescu theorem concerning limiting embeddings of fractional Sobolev spaces”. J. Funct. Anal. 201 (2003), 298-300.

[24] M. Milman, Notes on limits of Sobolev spaces and the continuity of interpolation scales. Trans. Amer. Math. Soc. 357 (2005), 3425-3442.

[25] M. Milman, BMO: oscillations, self improvement. Gagliardo coordinate spaces and reverse Hardy inequalities. Preprint, 2015, http://arxiv.org/pdf/1505.02633.pdf .

[26] D. Schymura, An upper bound on the volume of the symmetric difference of a body and a congruent copy. Adv. Geom. 14 (2014), 287-298. 
[27] P. Silvestre, Capacities and embeddings via symmetrization and conductor inequalities. Proc. Amer. Math. Soc. 142 (2014), 497-505.

[28] J. Simon, Sobolev, Besov and Nikolskii fractional spaces: imbeddings and comparisons for vector valued spaces on an interval. Annali di Matematica pura ed applicata (IV), Vol. LCVII (1990), 117-148.

[29] H. Triebel, Limits of Besov norms. Arch. Math. 96 (2011), 169-175.

[30] R. Strichartz, Bounded mean oscillation and Sobolev spaces. Indiana Univ. Math. J. 29 (1980), $539-558$.

[31] Z. Wu, Strong type estimate and Carleson measures for Lipschitz spaces. Proc. Amer. Math. Soc. 127 (1999), 3243-3249.

[32] J. Xiao, Homogeneous endpoint Besov space embeddings by Hausdorff capacity and heat equation. Adv. Math. 207 (2006), 828-846.

[33] J. Xiao, Gaussian BV capacity, Adv. Cal. Var. 9(2016)187-200.

[34] J. Xiao, Optimal geometric estimates for fractional Sobolev capacities. C. R. Acad. Sci. Ser. I 354(2016)149-153.

[35] J. Xiao and D. Ye, Anisotropic Sobolev capacity with fractional order. Canadian J. Math. http://dx.doi.org/10.4153/CJM-2015-060-3.

[36] J. Xiao and Z. Zhai, C.S.I. for Besov spaces $\dot{\Lambda}_{\alpha}^{p, q}\left(\mathbb{R}^{n}\right)$ with $(\alpha,(p, q)) \in(0,1) \times((0,1] \times(0,1] \backslash\{(1,1)\})$. Some Topics in Harmonic Analysis and Applications, ALM 34, pp.405-417, Higher Education Press and International Press Beijing-Boston, 2015.

[37] W. Ziemer, Weakly Differentiable Functions. Springer Verlag, New York, 1989.

Instituto Argentino de Matematica, Buenos Aires, Argentina

E-mail address: mario.milman@gmail.com

Department of Mathematics and Statistics, Memorial University, St. John's, NL A1C5S7, Canada

E-mail address: jxiao@mun.ca 